\title{
Spectroscopic aperture biases in inside-out evolving early-type galaxies from CALIFA ${ }^{\star}$
}

\author{
J. M. Gomes ${ }^{1}$, P. Papaderos ${ }^{1}$, J. M. Vílchez ${ }^{2}$, C. Kehrig ${ }^{2}$, J. Iglesias-Páramo ${ }^{2,3}$, I. Breda ${ }^{1}$, M. D. Lehnert ${ }^{4}$, \\ S. F. Sánchez ${ }^{5}$, B. Ziegler ${ }^{6}$, S. N. dos Reis ${ }^{1}$, J. Bland-Hawthorn ${ }^{7}$, L. Galbany ${ }^{8,9}$, D. J. Bomans ${ }^{10,11}$, \\ F. F. Rosales-Ortega ${ }^{12}$, C. J. Walcher ${ }^{13}$, R. García-Benito ${ }^{2}$, I. Márquez ${ }^{2}$, A. del Olmo ${ }^{2}$, M. Mollá ${ }^{14}$, R. A. Marino ${ }^{15,16}$, \\ C. Catalán-Torrecilla ${ }^{17}$, R. M. González Delgado², Á. R. López-Sánchez ${ }^{18,19}$, and the CALIFA collaboration \\ (Affiliations can be found after the references)
}

Received 5 September 2015 / Accepted 2 November 2015

\begin{abstract}
Integral field spectroscopy (IFS) studies based on CALIFA survey data have recently revealed ongoing low-level star formation (SF) in the periphery of a small fraction $(\sim 10 \%)$ of local early-type galaxies (ETGs), witnessing a still ongoing inside-out galaxy growth process. A distinctive property of the nebular component in these ETGs, classified i+, is a structure with two radial zones, the inner of which displays LINER emission with a $\mathrm{H} \alpha$ equivalent width $E W(\mathrm{H} \alpha) \simeq 1 \AA$, the outer $(3 \AA<E W(\mathrm{H} \alpha) \lesssim 20 \AA)$ HII-region characteristics. Using CALIFA IFS data, we empirically demonstrate that the confinement of nebular emission to the galaxy periphery leads to a strong aperture (or, correspondingly, redshift) bias in spectroscopic single-fiber studies of type i+ ETGs: at low redshift $(z \lesssim 0.45)$, SDSS spectroscopy is restricted to the inner (SF-devoid LINER) zone, which causes the galaxies to be erroneously classified as "retired", that is, systems entirely lacking SF, and whose faint nebular emission is solely powered by the post-AGB stellar component. The SDSS aperture progressively encompasses the outer SF zone only at higher $z$, at which the galaxies are unambiguously classified as "composite SF/LINER". We also empirically demonstrate that the principal effect of a decreasing spectroscopic aperture on the classification of $\mathrm{i}+\mathrm{ETGs}$ through standard $[\mathrm{NII}] / \mathrm{H} \alpha$ vs. [OIII]/H $\beta$ emission-line (BPT) ratios consists of a monotonic shift upward and to the right precisely along the upper right wing of the "seagull" distribution on the BPT plane, that is, along the pathway connecting composite SF/HII galaxies with AGN/LINERs. Motivated by these observational insights, we also investigate theoretically observational biases in aperture-limited studies of inside-out growing galaxies as a function of $z$. To this end, we devise a simple 1D model that involves an outward-propagating exponentially decreasing SF process since $z \sim 10$ and reproduces the radial extent and two-zone $E W(\mathrm{H} \alpha)$ distribution of local i+ ETGs. By simulating the 3" spectroscopic SDSS aperture in this model, we find that SDSS studies at $z \lesssim 1$ are progressively restricted to the inner (SF-devoid LINER) zone and miss an increasingly larger portion of the $\mathrm{H} \alpha$-emitting periphery. This leads to the incorrect spectroscopic classification of these inside-out assembling galaxies as retired ETG/LINERs and also to a severe underestimation of their total star formation rate (SFR) in a manner inversely related to $z$. More specifically, the SFR inferred from the H $\alpha$ luminosity registered within the SDSS fiber is reduced by $50 \%$ at $z \sim 0.86$, reaching only $0.1 \%$ of its integral value at $z=0.1$. We argue that the aperture-driven biases described above pertain to any morphological analog of i+ ETGs (e.g., SF-quiescent bulges within star-forming disks), regardless of whether it is viewed from the perspective of inside-out growth or inside-out SF quenching, and might be of considerable relevance to galaxy taxonomy and studies of the cosmic SFR density as a function of $z$
\end{abstract}

Key words. galaxies: elliptical and lenticular, cD - galaxies: nuclei - galaxies: ISM - galaxies: star formation

\section{Introduction}

Studies of large extragalactic samples with single-fiber spectroscopy from the SDSS (York et al. 2000) and GAMA (Driver et al. 2009) have largely been relying on the assumption that aperture-effects are negligible or can be accounted for in a statistical sense. For example, Kewley et al. (2005) reported that the condition of the $3^{\prime \prime}$ SDSS fiber enclosing $\sim 20 \%$ of the total emission of a galaxy suffices for minimizing systematic and random aperture-related errors, and recommended selecting samples at redshifts $z>0.04$. More generally, numerous singlefiber studies (e.g., Kauffmann et al. 2003; Brinchmann et al. 2004; Tremonti et al. 2004) are based on the assumption that the spectroscopic aperture encompasses a representative probe of the spectrum of a galaxy, thus astrophysical quantities derivable

* Based on observations collected at the Centro Astronómico Hispano Alemán (CAHA) at Calar Alto, operated jointly by the MaxPlanck-Institut für Astronomie (MPIA) and the Instituto de Astrofísica de Andalucía (CSIC). from it (e.g., the $\mathrm{H} \alpha$ luminosity) are either characteristic for a galaxy as a whole or can be converted into integral ones through simple parametrizations.

This rationale essentially permeates the entire work that has dealt with large extragalactic probes and their evolution with $z$ in the era of SDSS and GAMA, and has gone a long way in our current understanding of a wide range of topical issues, for example, related to the dependence of the star formation rate (SFR) and specific SFR on stellar mass $\left(\mathcal{M}_{\star}\right)$, the cosmic evolution of the SFR density, and the spectral galaxy classification on the basis of diagnostic emission-line ratios (e.g., $[\mathrm{N} \mathrm{II}] 6584 / \mathrm{H} \alpha$ vs. [O III $] 5007 / \mathrm{H} \beta$, Baldwin et al. 1981, hereafter BPT). For instance, determinations of integral SFRs have by necessity had to rely on an extrapolation of emission-line measurements within the spectroscopic aperture assuming that the $\mathrm{H} \alpha$ scales with the underlying stellar continuum throughout the galaxy extent or is linked to broad-band colors. For example, Hopkins et al. (2003) estimated integral SFRs from the $\mathrm{H} \alpha$ luminosity within the spectroscopic aperture and assumed 
a constant equivalent width $(E W(\mathrm{H} \alpha))$ throughout the galaxy extent. Similarly, Brinchmann et al. (2004) employed a probabilistic estimate for the total SFR of SDSS galaxies that relates broad-band colors with the $\mathrm{H} \alpha$ luminosity. An inherent weakness of these approaches obviously is that, depending on the $z$ and the linear extent of a galaxy, the fiber spectrum can be biased toward particular luminosity entities (e.g., the brightest starforming knot of a starburst galaxy or the non-star-forming bulge of a late-type star-forming disk), making an extrapolation to integral quantities uncertain.

The advent of integral field spectroscopy (IFS) over a large field of view (FoV), for example, from the Calar Alto Legacy Integral Field Area survey (CALIFA, Sánchez et al. 2012), has recently permitted empirical studies of aperture-effects on various physical properties of the nebular emission in late-type galaxies (for instance, $\mathrm{H} \alpha$ luminosity and equivalent width $\mathrm{EW}$; e.g., Gerssen et al. 2012; Iglesias-Páramo et al. 2013; Brough et al. 2013; Belfiore et al. 2015).

This subject has not been investigated in similar detail for early-type galaxies (ETGs), however, partly because of the faintness of their nebular emission and the associated uncertainties in studying them, and presumably also due to the widespread view that these systems are comparatively simple and spatially homogeneous in the characteristics of their stellar and nebular components. This picture has now been substantially revised through IFS studies, which continue to reveal an impressively high complexity in both (e.g., Sarzi et al. 2006; McDermid et al. 2007; Sarzi et al. 2010; Krajnović et al. 2011; Kehrig et al. 2012; Arnold et al. 2014; Houghton et al. 2013; Pracy et al. 2014; Gomes et al. 2015a, hereafter G15a), with conspicuous radial trends in stellar age (e.g., Pérez et al. 2013; González Delgado et al. 2014, G15a) and the $E W(\mathrm{H} \alpha)$ of local ETGs (G15a, Papaderos et al. 2013, hereafter P13). It appears to be especially important for a better understanding of aperture effects on spectroscopic BPT classifications of ETGs to investigate the gas excitation mechanism in a spatially resolved manner with IFS data. Several studies over the past years have pointed out the role of low-level SF activity (see, e.g., Trager et al. 2000; Schawinski et al. 2007; Shapiro et al. 2010, and references therein) on the excitation of faint nebular emission in ETGs, as an alternative or supplementary mechanism to photoionization by an active galactic nucleus (AGN; e.g., Ho 2008) or the evolved ( $\geq 10^{8} \mathrm{yr}$ ) postAGB (pAGB) population (e.g., Trinchieri \& di Serego Alighieri 1991; Binette et al. 1994; Stasińska et al. 2008), or fast shocks (e.g., Dopita \& Sutherland 1995). Observational evidence for SF in ETGs has been accumulating from multi-wavelength studies (e.g., Kaviraj et al. 2007; Gil de Paz et al. 2007; Schawinski et al. 2009; Huang \& Gu 2009; Salim et al. 2012; Petty et al. 2013; Ko et al. 2014; Pan et al. 2014). For example, Kaviraj et al. (2008) found that $10-15 \%$ of the stellar mass $\left(\mathcal{M}_{\star}\right)$ in these systems has been built in a declining SF process since $z \simeq 1$, a conclusion that appears to be in line with the small fraction $(\sim 5.7 \%)$ of blue ETGs in the local Universe with estimated SF rates (SFRs) of between 0.5 and $50 M_{\odot} / \mathrm{yr}$ (Schawinski et al. 2009). It is also noteworthy in this context that spatially resolved studies of individual ETGs indicate an outward-increasing luminosity contribution from young-to-intermediate-age stellar populations (e.g., Fang et al. 2012; Gomes et al. 2015a).

In particular, Gomes et al. (2015a, see also G15b) identified a small fraction $(\sim 10 \%)$ of CALIFA ETGs (classified as type $\mathrm{i}+$ ) that show a steep $E W(\mathrm{H} \alpha)$ increase in their periphery. As demonstrated in a subsequent study (Gomes et al. 2016, hereafter G16), this outer $E W(\mathrm{H} \alpha)$ excess is due to $\mathrm{SF}$, reflecting a still ongoing inside-out galaxy buildup process.
Central to our considerations in this study is the fact that the dominant fraction $(60-80 \%)$ of the total $\mathrm{H} \alpha$ emission in all but one i+ ETGs studied in G16 arises beyond one effective radius $R_{\text {eff }}\left(\geq 10^{\prime \prime}\right)$, it thus evades detection within the $3^{\prime \prime}$ SDSS aperture. Our aim here is to explore aperture-effects on spectroscopic SDSS studies of type i+ ETGs and other inside-out forming galaxies as a function of cosmic time. To this end, we take a twofold approach that combines an empirical assessment of aperture biases using as templates CALIFA IFS data for the galaxies studied in G16 (Sect. 2) with simulations on the basis of a simplified inside-out galaxy growth model (Sect. 3). Our conclusions are summarized in Sect. 4.

\section{Anatomy of type $i+E T G s$ and aperture biases on their spectroscopic classification}

The defining characteristics of i+ ETGs were discussed in G15a-c on the basis of three CALIFA galaxies (Fig. 1). A distinctive property of the nebular component of these systems is a structure with two radial zones, the inner of which contains faint $(E W(\mathrm{H} \alpha) \simeq 1 \AA)$ LINER emission, the outer $(3 \AA<E W(\mathrm{H} \alpha) \lesssim 20 \AA)$ essentially shows HII-region characteristics.

This two-zone morphology can be best illustrated in the case of NGC 1349: Its central part $\left(R^{\star} \leq 8^{\prime \prime}\right.$, equivalent to $\left.3.4 \mathrm{kpc}\right)$ shows a low, nearly constant $E W(\mathrm{H} \alpha)$ of $1 \AA$, which is consistent with gas photoionization by the evolved $\left(\geq 10^{8} \mathrm{yr}\right)$ post-AGB stellar component (cf., e.g., G15a), whereas the high $(\sim 10 \AA)$ $E W(\mathrm{H} \alpha)$ and BPT ratios in its periphery $\left(R^{\star} \geq 8^{\prime \prime}\right)$ imply gas photoionization by massive OB stars. Using the isophotal annuli technique (Papaderos et al. 2002, P13), we extracted the spectrum within the inner and outer zone, which are shown with the integrated spectrum overlaid in Fig. 1a. The middle panel shows the pure nebular component in the region around the $\mathrm{H} \alpha$ Balmer line as obtained after subtracting the best-fitting stellar model (see G15a for details). This diagram shows the significant decrease in $[\mathrm{N} \mathrm{II}] / \mathrm{H} \alpha$ flux ratio from the inner to the outer zone. This effect can be better evaluated in panel c, where we illustrate the change in position of the type i+ ETGs on the BPT plane when the galaxy spectrum is sampled within successively larger annuli: Including the very faint SF rim for NGC 1167 has a marginal effect on its classification as an AGN/LINER, whereas in NGC 1349 the much brighter peripheral SF zone results in a shift downward and to the left below the LINER demarcation curve, moving the ETG into the locus of "composite SF/LINER" galaxies. Had this galaxy been observed within a FoV of $\emptyset \leq 20^{\prime \prime}$, then only its central, almost emission-line free zone would have been considered in the analysis, prompting its spectroscopic classification as a retired ETG/LINER in the definition by Stasińska et al. (2008). Obviously, the same conclusion would have been drawn from SDSS or GAMA spectra (York et al. 2000; Baldry et al. 2010). The aperture of these surveys would encompass the whole ETG only beyond $z \gtrsim 0.45$, where its true nature is revealed. In the specific context of i+ ETGs (i.e., systems where SF is mainly confined to the galaxy periphery), the decreasing (increasing) proportion of star-forming (retired) galaxies with decreasing redshift (e.g., Stasińska et al. 2015) could therefore be partly driven by aperture effects.

In summary, a decrease in the spectroscopic aperture can result in the case of i+ ETGs in a shift upward and to the right precisely along the right wing of the "seagull" distribution on the BPT plane, that is, along the pathway connecting SF/HII galaxies with AGN/LINERs. This empirical fact calls for a critical 
J. M. Gomes et al.: Spectroscopic aperture biases in inside-out evolving early-type galaxies from CALIFA
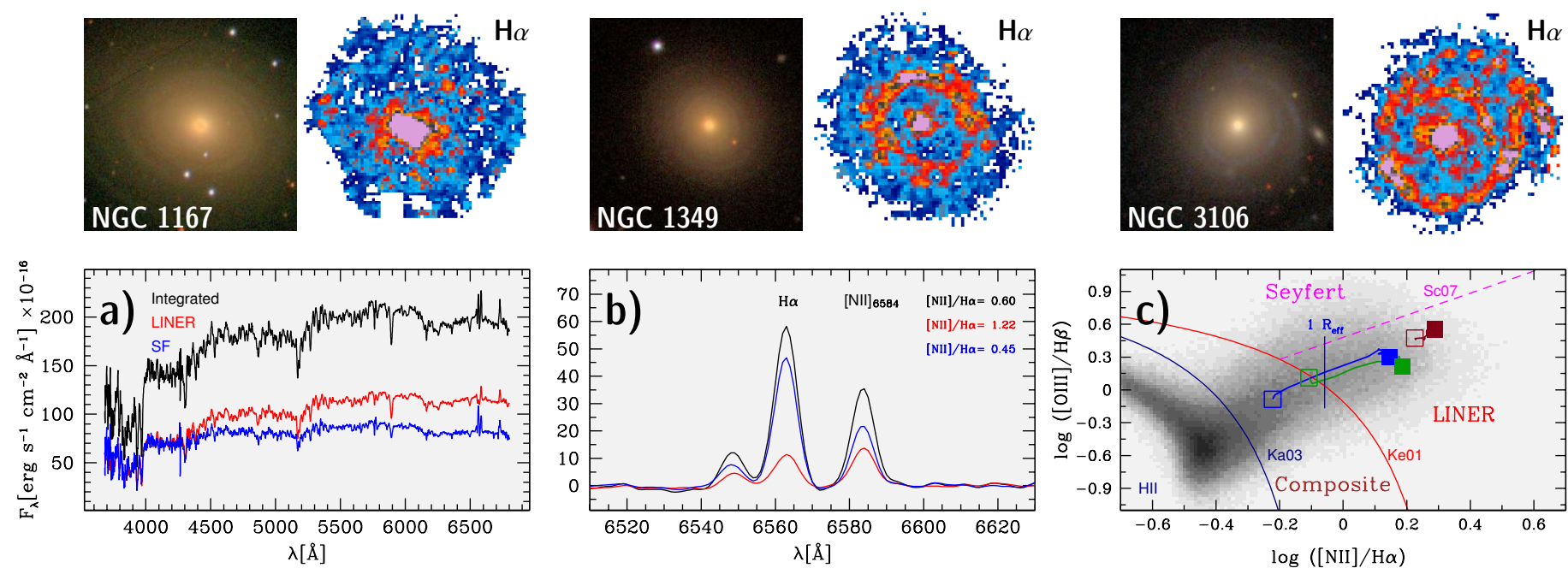

Fig. 1. Upper panels: SDSS true-color images and $\mathrm{H} \alpha$ maps (displayed between 0.05 and $1 \times 10^{-16} \mathrm{erg} \mathrm{s}^{-1} \mathrm{~cm}^{-2}$ ) of the three i+ ETGs (NGC 1167 , NGC 1349 and NGC 3106; from left to right) studied in Gomes et al. (2015b, 2016). Lower panels: a) comparison of the integrated spectrum of NGC 1349 within its inner $\left(R^{\star} \leq 8^{\prime \prime}\right)$ LINER zone (red) with that obtained from its outer $\left(R^{\star}>8^{\prime \prime}\right)$ star-forming zone (blue). The integrated spectrum of the ETG is overlaid in black. b) Zoom-in into the pure nebular spectrum of NGC 1349 around the H $\alpha 6563 \AA$ Balmer line, as obtained after subtracting the best-fitting stellar model from the observed spectrum (see P13 and G15a for details). The color coding is the same as in panel a. Note the change of the $[\mathrm{N} \mathrm{II}] / \mathrm{H} \alpha$ emission-line flux ratio from the inner (LINER) to the outer (SF-dominated) zone. c) Variation of the diagnostic BPT ratios for the three $\mathrm{i}+$ ETGs as a function of the aperture considered in their analysis. Brown, blue, and green correspond to NGC 1167, NGC 1349, and NGC 3106, respectively. The nuclear BPT ratios and those determined from the integrated galaxy spectra are shown with filled and open squares, respectively, and the connecting lines mark determinations based on successively larger spectroscopic apertures. The equivalent aperture radius in $R_{\text {eff }}$ is indicated for NGC 1349 only for the sake of clarity. Note the shift of NGC 1349 from the LINER into the "composite SF/LINER" regime of the BPT diagram when instead of the inner zone $\left(\leqslant 0.7 R_{\text {eff }}\right)$ the integral spectrum of the ETG is considered. The shaded background depicts the surface density of galaxies from SDSS in the upper right wing of the BPT plane. The overlaid curves show the demarcation between AGN and LINERs (Schawinski et al. 2007, SC07), the locus of HII regions (Kauffmann et al. 2003, Ka03), and the "maximum SF" boundary (Kewley et al. 2001, Ke01).

reconsideration of the way this right wing should be interpreted in addressing the relative role of thermal and non-thermal activity in i+ ETGs and their morphological analogs (e.g., late-type galaxies with an old, SF-devoid bulge within a more extended star-forming disk).

\section{Inside-out galaxy formation and associated aperture effects}

Motivated by the observational evidence laid out in G16 and in the previous section, we extend our study by considering an inside-out formation process (e.g., Pérez et al. 2013; González Delgado et al. 2014) for ETGs. This is simulated by an outward-propagating SF process in a 1D galaxy model, which, combined with an evolutionary synthesis code (cf. G15a), permits computing the time evolution of various spectrophotometric properties as a function of radius.

A SF propagation process with a velocity $v$ can be written by making use of the $1 \mathrm{D}$ wave equation:

$\nabla^{2} \operatorname{SFR}(r, t)=\frac{\partial^{2} \operatorname{SFR}(r, t)}{\partial r^{2}}=\frac{1}{v^{2}} \frac{\partial^{2} \operatorname{SFR}(r, t)}{\partial t^{2}}$,

where the solution of this partial differential equation is given by D'Alembert (1747) with a linear sum of two arbitrary functions $f(v t-r)$ and $g(v t+r)$ that represent an incoming and outgoing wave, respectively. Since the SFR wave propagates outward as a result of the inside-out growth of the galaxy, we can write the general solution as $\operatorname{SFR}(r, t)=f(v t-r)=\phi(t-r / v)$.

For each radial zone, the $\operatorname{SFR}(r, t)$ is chosen to be exponentially declining as $\propto e^{-(t-r / v) / \tau}$ with an e-folding timescale of $\tau=1 \mathrm{Gyr}$, constant wave velocity $v$, and subject to the constraint $t \geq r / v$. This scenario is compatible with a quick cessation of SF in the inner zone (as expected, e.g., during the bulge formation) and continued residual SF in the galaxy periphery in an inside-out galaxy buildup process. For simplicity, the galaxy is constructed such as to display a constant age gradient $\nabla t=-0.5 \mathrm{Gyr} / \mathrm{kpc}$, which imposes a maximum radius as a function of lookback time of $R_{\max }(t)=\left(t_{0}-t\right) /|\nabla t|$, where $t_{0}$ is the galaxy formation lookback time. For the adopted model, the galaxy grows radially at a constant speed $v \sim 2 \mathrm{~km} \mathrm{~s}^{-1}$, and when it attains $R_{\max }=20 \mathrm{kpc}$ (at $z \sim 0.3$ ), the linear growth ceases, yielding a typical present-day ETG radius (e.g., Gomes et al. 2015a). The Friedmann-Robertson-Walker cosmology with $H_{0}=70 \mathrm{~km} \mathrm{~s}^{-1} \mathrm{Mpc}^{-1}, \Omega_{\mathrm{m}}=0.3$ and $\Omega_{\Lambda}=0.7$ was adopted, yielding $13.52 \mathrm{Gyr}$ for the age for the Universe. The time $t_{0}$ of galaxy formation is taken to be $\sim 0.5 \mathrm{Gyr}$ (redshift 10) after the Big Bang.

The spectral energy distribution (SED) is computed following the detailed prescriptions by G15a. The synthetic composite stellar SEDs were evaluated using the full set of ages for the simple stellar populations (SSPs) from Bruzual \& Charlot (2003, hereafter BC03); they comprise 200 spectra spanning an age between 0 and $13.52 \mathrm{Gyr}$ and assume a constant solar metallicity. We note that even though emission-line luminosities and their ratios for subsolar metallicities can significantly change due to the harder, increased UV ionizing output of massive lowmetallicity stars, the principal trends and conclusions inferred from the adopted inside-out galaxy growth model remain unaltered. The BC03 SSP library uses the Padova 1994 evolutionary tracks (Alongi et al. 1993; Bressan et al. 1993; Fagotto et al. 1994a,b,c; Girardi et al. 1996), the Chabrier (2003) initial mass function, and the STELIB stellar library (Le Borgne et al. 2003). 

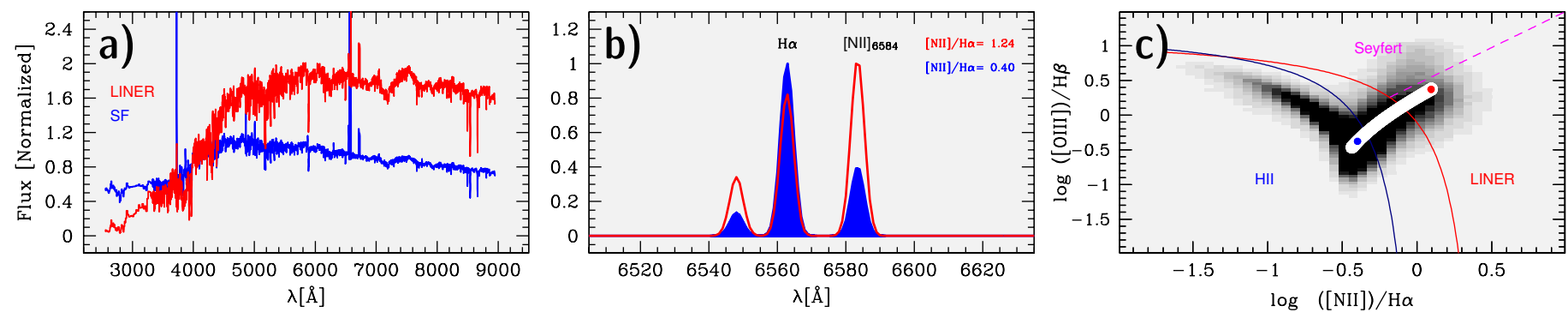

Fig. 2. Simulation of spectroscopic aperture bias for an inside-out formed galaxy according to the 1D model described in Sect.3 (see Fig. 3 ) that is observed with SDSS at a redshift $z=0.1$. At this $z$ the SDSS fiber $\left(\emptyset=3^{\prime \prime}\right)$ covers a projected linear diameter of $\sim 5.5 \mathrm{kpc}$. a) Comparison between the integrated spectrum of our model galaxy (blue) with that obtained within the SDSS fiber (red). Both spectra are normalized at $4020 \AA$. b) Zoom-in into the spectral region around the $\mathrm{H} \alpha$ line after removing the underlying stellar continuum. The color coding is the same as in panel a. Note the considerable difference in the $[\mathrm{N} \mathrm{II}] / \mathrm{H} \alpha$ emission-line ratio from the integral and simulated SDSS spectrum of the galaxy. From the former, the galaxy would be spectroscopically classifiable as a star-forming galaxy, while the latter would imply a purely retired LINER galaxy. c) Variation of the BPT line ratios as a function of increasing aperture size for the adopted galaxy model (white). The simulated ratios form a continuous sequence along the right wing of the BPT diagram, moving from the LINER toward the HII zone as the aperture size increases, in close resemblance to the observational trend seen in Fig. 1c. The $[\mathrm{N} \mathrm{II}] / \mathrm{H} \alpha$ and $[\mathrm{O}$ III $] / \mathrm{H} \beta$ ratios for the integrated and the simulated SDSS spectrum are shown in blue and red, respectively. The gray shaded background depicts the surface density of galaxies from SDSS, and the overlaid curves are the same as in Fig. 1c.

Hydrogen (Balmer, Paschen, etc.) line fluxes were computed from the total UV ionizing flux assuming case B recombination $\left(T_{\mathrm{e}}=10000 \mathrm{~K}\right.$ and $\left.n_{\mathrm{e}}=100 \mathrm{~cm}^{-3}\right)$ with the corresponding effective recombination coefficient $\alpha_{\mathrm{H} \alpha}^{\mathrm{eff}}$ and by assuming ionization-bound nebulae. The fluxes of collisionally excited lines are based on semi-empirical calibrations for a) classical HII regions (e.g., Anders \& Fritze 2003), photoionized by OB stars, and b) ETG nuclei that are photoionized by post-AGB stars (old stellar populations $\geq 1 \mathrm{Myr}$ ) and show LINER BPT ratios (e.g., Binette et al. 1994). Therefore, the total UV ionizing flux from an exponentially declining SFR model with $\tau=1 \mathrm{Gyr}$ contains the combined output from a young SF plus an old postAGB component in certain evolutionary phases that produces a luminosity-weighted average of the modeled emission lines.

The computed radial profiles for the $E W(\mathrm{H} \alpha)$ and lightweighted stellar age for various evolutionary stages (from 0 to 13.52 Gyr) are shown in Fig. 3. It is apparent that the $E W(\mathrm{H} \alpha)$, in general, decreases as a function of time, while the stellar age always increases.

The $3^{\prime \prime}$ SDSS aperture was subsequently simulated on the model galaxy to evaluate aperture effects and their dependence on $z$. In the adopted cosmological model, the linear radius projected within the SDSS fiber as a function of $z$ was simulated on the model (Fig. 4). For $z \lesssim 1$ the SDSS aperture encompasses a progressively smaller fraction of the galaxy. Quite importantly, the fraction of $\mathrm{H} \alpha$ luminosity registered within the SDSS fiber (upper panel) has a much steeper decline than the fractional area covered by SDSS because the SF activity is confined to the galaxy periphery. Quantitatively, the $\mathrm{H} \alpha$ flux registered within the SDSS fiber decreases by $50 \%$ at $z \sim 0.86$, reaching only $0.1 \%$ of its integral value at $z=0.1^{1}$.

Consequently, a strong aperture bias is to be expected in SFR determinations and the spectroscopic classification of inside-out assembling $\mathrm{i}+$ ETGs and their morphological analogs. To exemplify this bias, a snapshot at $z \sim 0.1$ is shown in Fig. 2. At this stage, in which the model galaxy has reached its maximum radius, the SDSS fiber samples a projected radius of $\sim 2.75 \mathrm{kpc}$

\footnotetext{
1 The fact that in i+ ETGs (and any inside-out forming galaxy in general) nebular emission is confined to the galaxy periphery and almost absent in the central part reinforces the warning voiced by Salim et al. (2012) who pointed out that the absence of $\mathrm{H} \alpha$ in SDSS spectra serves as a reminder that the information from fiber spectra cannot always be extrapolated to entire galaxies.
}
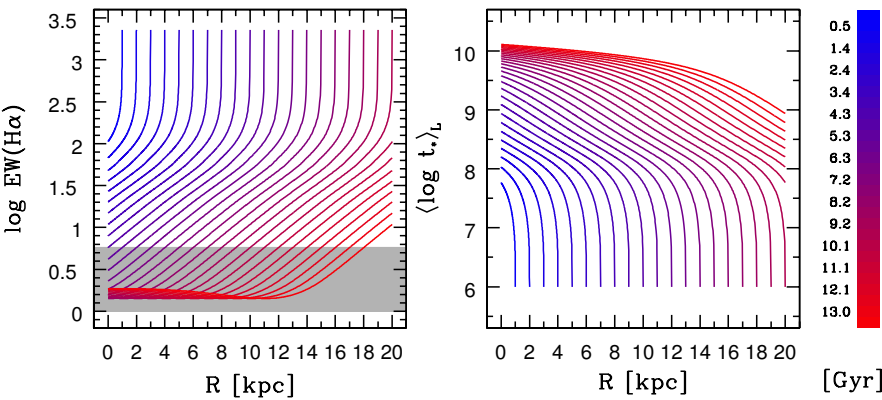

Fig. 3. Radial distribution of the $E W(\mathrm{H} \alpha)$ and the light-weighted mean stellar age $\left(\left\langle\log t_{\star}\right\rangle_{L}\right)$, as predicted by the adopted inside-out galaxy formation scenario for 28 ages between 0 and $13.52 \mathrm{Gyr}$ (cf. color coding on the right side bar).The region where the observed $E W(\mathrm{H} \alpha)$ is consistent with pure post-AGB photoionization $(<2.4 \AA)$ is shown with the shaded gray area (see G15a). The model adopts a constant age gradient $\nabla t=-0.5 \mathrm{Gyr} / \mathrm{kpc}$ and outward propagating SF at $v \sim 2 \mathrm{~km} \mathrm{~s}^{-1}$. In this particular simulation the inside-out growth ceases when the galaxy reaches a radius of $20 \mathrm{kpc}$. The observational properties are computed for each zone assuming an exponentially declining SFR with an efolding timescale of $1 \mathrm{Gyr}$.

( $\sim 2 \%$ of the area of the galaxy). As apparent from panel a, the integrated galaxy spectrum is very different from the one registered within the SDSS fiber: The former is characteristic of a blue star-forming galaxy, showing BPT ratios typical of HII regions, whereas the latter indicates a retired LINER/ETG.

This is also reflected in the BPT diagram (panel c), where the white curve delineates the variation of the BPT line-ratios obtained for our model galaxy within a set of increasing apertures. The $[\mathrm{N} \mathrm{II}] / \mathrm{H} \alpha$ and $[\mathrm{O}$ III $] / \mathrm{H} \beta$ ratios corresponding to the integrated spectrum and those registered within the SDSS aperture are shown as blue $(0.40,0.42)$ and red $(1.24,2.36)$ dots, respectively. The simulated BPT ratios describe a continuous sequence along the right wing of SDSS determinations (shaded area), connecting the LINER with the HII/SF zone of the BPT parameter space, in agreement with the observational trend described in Sect. 2 (Fig. 1c). Especially important in this context is that the identical trend arises when, instead of using successively larger apertures at $z=0.1$, the SDSS aperture is simulated on the inside-out evolving galaxy across $z$. 
J. M. Gomes et al.: Spectroscopic aperture biases in inside-out evolving early-type galaxies from CALIFA

Lookback Time [Gyr]

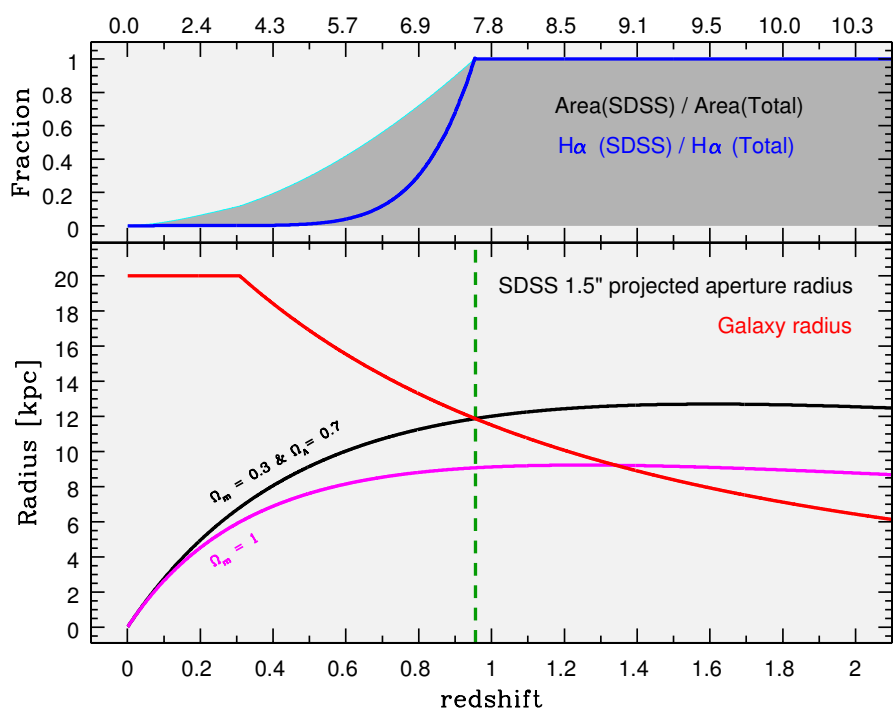

Fig. 4. Top panel: fraction of the area (shaded region) and of the total $\mathrm{H} \alpha$ flux (blue curve) encompassed by the $3^{\prime \prime}$ SDSS fiber as a function of $z$ (or, equivalently, lookback time in Gyr; upper label). Bottom panel: radius projected within the SDSS fiber as a function of $z$ for a Friedmann-Robertson-Walker cosmology with $H_{0}=70 \mathrm{~km} \mathrm{~s}^{-1} \mathrm{Mpc}^{-1}$, $\Omega_{\mathrm{m}}=0.3$ and $\Omega_{\Lambda}=0.7$ (black curve) and, for the sake of comparison, a flat, matter-dominated Universe $\left(\Omega_{\mathrm{m}}=1\right.$; magenta curve). The radius of the inside-out forming galaxy is drawn in red and the dashed line marks the $z=0.95$ below which the area subtended by the SDSS fiber becomes smaller than the galaxy.

In summary, for an inside-out assembling galaxy with the model assumption adopted here, the simultaneous linear and angular growth of the inner (SF devoid/LINER) zone will cause limited-aperture surveys to strongly underestimate the total SFR in a manner inversely related to $z$. This bias, arising at $z \lesssim 1$ and becoming progressively severer for lower $z$, could artificially increase the estimated fraction of non-star-forming (retired) galaxies over the past $\sim 7 \mathrm{Gyr}$, which would affect the galaxy classification and mimic a steeper decline of the cosmic SFR density.

\section{Summary and conclusions}

This study has been motivated by the recent detection of faint spiral-like features in the low-surface brightness periphery of a small subset $(\sim 10 \%)$ of nearby early-type galaxies (ETGs) that was reported by Gomes et al. $(2015 \mathrm{a}, \mathrm{b})$ from the CALIFA integral field spectroscopic (IFS) galaxy survey. As a subsequent analysis by Gomes et al. (2016) has revealed, these features witness a still ongoing inside-out galaxy growth process and are spatially associated with an extended emission-line zone containing up to $\sim 80 \%$ of the total $\mathrm{H} \alpha$ emission of these galaxies. A distinctive property of the nebular component in these ETGs, classified as $\mathrm{i}+$, is a structure with two radial zones, the inner of which is on the order of the galaxy effective radius $R_{\text {eff }}\left(\sim 10^{\prime \prime}\right)$ and contains faint $(E W(\mathrm{H} \alpha) \simeq 1 \AA)$ LINER emission, the outer $(3 \AA<E W(\mathrm{H} \alpha) \lesssim 20 \AA)$ displays HII-region characteristics.

The question that naturally arises from the observed segregation of the nebular emission in i+ ETGs in two spatially and physically distinct concentric zones is how aperture effects might impact spectroscopic studies of these systems. To address these questions, we here adopted a combined empirical and theoretical approach, aiming at a qualitative assessment of aperture-driven biases in SDSS studies of type i+ ETGs and other inside-out forming galaxies across cosmic time.

In the first stage, we empirically demonstrated with CALIFA IFS data that for a typical i+ ETG the confinement of nebular emission to the galaxy periphery leads to a strong observational bias in spectroscopic studies with SDSS. At low redshift $(z \lesssim 0.45)$, the $3^{\prime \prime}$ SDSS fiber only captures the inner (non-star-forming LINER) zone of such a galaxy, which causes it to be erroneously classified as retired, that is, as a system entirely lacking ongoing star formation (SF) and one whose faint nebular emission is solely powered by the post-AGB stellar component. The SDSS aperture progressively encompasses the outer (star-forming) zone only at higher $z$, where it can be unambiguously classified as a "composite SF/LINER". We also empirically demonstrated that the principal effect of a decreasing spectroscopic aperture on the classification of $i+$ ETGs through standard $[\mathrm{NII}] / \mathrm{H} \alpha$ vs. [OIII] $/ \mathrm{H} \beta$ emission-line (BPT) ratios consists of a monotonic shift upward and to the right precisely along the right wing of the "seagull" distribution on the BPT plane, that is, along the pathway connecting composite $\mathrm{SF} / \mathrm{HII}$ galaxies with AGN/LINERs.

These empirical insights were further underscored through a simple 1D inside-out galaxy formation model involving an outward-propagating exponentially decreasing SF process since $z=10$, which reproduces both the radial extent and the two-zone $E W(\mathrm{H} \alpha)$ morphology of present-day i+ ETGs. By simulating the SDSS aperture in this model, we found that for $z \lesssim 1$, SDSS spectroscopy is progressively restricted to the inner (SF-devoid LINER) zone of an inside-out forming galaxy, thereby missing an increasingly larger portion of its $\mathrm{H} \alpha$-emitting periphery. More specifically, according to our model, the $\mathrm{H} \alpha$ flux registered within the SDSS fiber decreases by $50 \%$ at $z \simeq 0.86$, reaching only $0.1 \%$ of its integral value at $z=0.1$.

For local $(z \simeq 0.1) \mathrm{i}+$ ETGs our model also reproduces the observed variation of BPT line-ratios with aperture sizes along the right wing of SDSS determinations, lending further support to the conjecture that spectroscopic classification on the basis of SDSS data is prone to substantial aperture effects. It is particularly important in this context that the same trend along the right wing is reproducible when the SDSS aperture is simulated on an inside-out forming galaxy since $z=10(\sim 13 \mathrm{Gyr})$ instead of using successively larger apertures for a local model-ETG.

The combined empirical and theoretical evidence from this study therefore suggests that the right wing distribution of BPT determinations for i+ ETGs and their morphological analogs (e.g., late-type galaxies with an old SF-devoid bulge centered on a more extended star-forming disk) with single-fiber spectroscopy (e.g., SDSS, GAMA) is consistent with (yet no proof for) a pure aperture effect and is naturally reproducible in an inside-out galaxy growth scenario. This calls for a closer examination of the way this right wing should be interpreted in addressing the relative role of thermal and non-thermal activity in these systems.

Finally, in the framework of the adopted inside-out galaxy formation model, the simultaneous linear and angular growth of the inner (SF devoid/LINER) zone will cause limited-aperture surveys (e.g., SDSS, GAMA) to strongly underestimate the total star formation rate (SFR) in a manner inversely related to $z$. This bias, arising at $z \lesssim 1$ and becoming progressively severer for lower $z$, could artificially increase the estimated fraction of non-star-forming (retired) galaxies over the past $\sim 7 \mathrm{Gyr}$, which would affect galaxy classification and mimic a steeper decline of the cosmic SFR density. 
These considerations underscore the critical importance of IFS studies of ETGs (and galaxies in general) over their entire optical extent.

Acknowledgements. This paper is based on data from the Calar Alto Legacy Integral Field Area Survey, CALIFA (http://califa.caha.es), funded by the Spanish Ministery of Science under grant ICTS-2009-10, and the Centro Astronómico Hispano-Alemán. JMG acknowledges support by Fundação para a Ciência e a Tecnologia (FCT) through the Fellowship SFRH/BPD/66958/2009 and POPH/FSE (EC) by FEDER funding through the program Programa Operacional de Factores de Competitividade (COMPETE). P.P. is supported by FCT through the Investigador FCT Contract No. IF/01220/2013 and POPH/FSE (EC) by FEDER funding through the program COMPETE. J.M.G., P.P., I.B. and S.N.d.R. acknowledge support by FCT under project FCOMP-01-0124FEDER-029170 (Reference FCT PTDC/FIS-AST/3214/2012), funded by FCTMEC (PIDDAC) and FEDER (COMPETE). They also acknowledge support by the exchange programme "Study of Emission-Line Galaxies with Integral-Field Spectroscopy" (SELGIFS, FP7-PEOPLE-2013-IRSES-612701), funded by the EU through the IRSES scheme. SFS acknowledges support from CONACyT-180125 and PAPIIT-IA100815 grants. Support for LG is provided by the Ministry of Economy, Development, and Tourism's Millennium Science Initiative through grant IC120009, awarded to The Millennium Institute of Astrophysics, MAS. L.G. acknowledges support by CONICYT through FONDECYT grant 3140566. C.J.W. acknowledges support through the Marie Curie Career Integration Grant 303912. I.M. acknowledges financial support by the Junta de Andalucía through project TIC114, and the Spanish Ministry of Economy and Competitiveness (MINECO) through projects AYA2010-15169 and AYA2013-42227-P. R.A. Marino is funded by the Spanish program of International Campus of Excellence Moncloa (CEI). This research made use of the NASA/IPAC Extragalactic Database (NED) which is operated by the Jet Propulsion Laboratory, California Institute of Technology, under contract with the National Aeronautics and Space Administration.

\section{References}

Alongi, M., Bertelli, G., Bressan, A., et al. 1993, A\&AS, 97, A851 Anders, P., \& Fritze-v. Alvensleben, U. 2003, A\&A, 401, 1063 Arnold, J. A., et al. 2014, ApJ, 791, A80

Baldry, I. K., Robotham, A. S. G., Hill, D. T., et al. 2010, MNRAS, 404, 86 Baldwin, J. A., Phillips, M. M., \& Terlevich, R. 1981, PASP, 93, 5 Belfiore, F., Maiolino, R., Bundy, K., et al. 2015, MNRAS, 449, 867 Binette, L., Magris, C. G., Stasińska, G., \& Bruzual, A. G. 1994, A\&A, 292, 13 Bressan, A., Fagotto, F., Bertelli, G., \& Chiosi, C. 1993, A\&AS, 100, 647 Brinchmann, J., Charlot, S., White, S. D. M., et al. 2004, MNRAS, 351, 1151 Brough, S., Croom, S., Sharp, R., et al. 2013, MNRAS, 435, 2903 Bruzual, G., \& Charlot, S. 2003, MNRAS, 344, 1000

Chabrier, G. 2003, ApJ, 586, L133

D'Alembert 1747, Suite des recherches sur la courbe que forme une corde tenduë mise en vibration, Histoire de l'académie royale des sciences et belles lettres de Berlin, 3, 220

Dopita, M. A., \& Sutherland, R. S. 1995, ApJ, 455, 468

Driver, S. P., Norberg, P., B., Ivan, K., et al. 2009, Astron. Geophys., 50, 12 Fagotto, F., Bressan, A., Bertelli, G., \& Chiosi, C. 1994a, A\&AS, 104, 365 Fagotto, F., Bressan, A., Bertelli, G., \& Chiosi, C. 1994b, A\&AS, 105, 29 Fagotto, F., Bressan, A., Bertelli, G., \& Chiosi, C. 1994c, A\&AS, 105, 39 Fang, Jerome J., Faber, S. M., et al. 2012, ApJ, 761, 23

Gerssen, J., Wilman, D. J., \& Cristensen, L. 2012, MNRAS, 420, 197 Gil de Paz, A., Boissier, S., Madore, B. F., et al. 2007, ApJS 173, 185 Girardi, L., Bressan, A., Chiosi, C., Bertelli, G., \& Nasi, E. 1996, A\&AS, 117, 113

Gomes, J. M., Papaderos, P., Kehrig, C., et al. 2015a, A\&A, in press, DOI: 10.1051/0004-6361/201525976, (G15a)

Gomes, J. M., Papaderos, P., Kehrig, C., et al. 2015b, in Galaxies in 3D across the Universe, eds. Ziegler et al., 105 (G15b)

Gomes, J. M., Papaderos, P., Vílchez, J. M., et al. 2016, A\&A, 585, A92, (G16)

González Delgado, R. M., Pérez, E., Cid Fernandes, R., et al. 2014, A\&A, 562, A47

Ho, L. C. 2008, ARA\&A, 46, 475

Hopkins, A. M., Miller, C. J., Nichol, R. C., et al., 2003, ApJ, 599, 971

Houghton, R. C. W., Davies, R. L., D’Eugenio, F., et al. 2013, MNRAS, 436, 19 Huang, S., \& Gu, Q. S. 2009, MNRAS, 398, 1651

Iglesias-Páramo, J., Vílchez, J. M., Galbany, L., et al. 2013, A\&A, 553, L7 Kauffmann, G., Heckman, T. M., Tremonti, C., et al. 2003, MNRAS, 346, 1055 Kaviraj, S., Schawinski, K., Devriendt, J. E. G., et al. 2007, ApJS, 173, 619 Kaviraj, S., Khochfar, S., Schawinski, K., et al. 2008, MNRAS, 388, 67
Kehrig, C., Monreal-Ibero, A., Papaderos, P., et al. 2012, A\&A, 540, A11 (K12) Kewley, L. J., Dopita, M. A., Sutherland, R. S., Heisler, C. A., \& Trevena, J. 2001, ApJ, 556, 121

Kewley, L. J., Jansen, R. A., \& Geller, M. J. 2005, PASP, 117, 227

Ko, J., Hwang, H. S., Im, M., et al. 2014, ApJ, 791, 134

Krajnović, D., Emsellem, E., Cappellari, M., et al. 2011, MNRAS, 414, 2923

Le Borgne, J.-F., Bruzual, G., Pelló, R., et al. 2003, A\&A, 402, L433

McDermid, R. M., Emsellem, E., Shapiro, K. L., et al. 2007, New Astron. Rev., 51,13

Pan, Z., Jinrong, L., Weipeng, L., et al. 2014, ApJ, 792, 1

Papaderos, P., Izotov, Y. I., Thuan, T. X., et al. 2002, A\&A, 393, 461

Papaderos, P., Gomes, J. M., Vílchez, J. M., et al. 2013, A\&A, 555, L1 (P13)

Pérez, E., Cid Fernandes, R., González Delgado, R. M., et al. 2013, ApJ, 764, L1

Petty, S. M., Neil, J. D., Jarrett, T. H., et al. 2013, AJ, 146, 4

Pracy, M. B., Owers, M. S., Zwaan, M., et al. 2014, MNRAS, 443, 388

Salim, S., Fang, J. J., Rich, R. M., et al. 2012, ApJ, 755, 2

Sánchez, S. F., Kennicutt, R. C., Gil de Paz, A., et al. 2012, A\&A, 538, A8 Sarzi, M., Falcón-Barroso, J., Davies, R. L., et al. 2006, MNRAS, 366, 1151

Sarzi, M., Shields, J. C., Schawinski, K., et al. 2010, MNRAS, 402, 2187

Schawinski, K., Thomas, D., Sarzi, M., et al. 2007, MNRAS, 382, 1415

Schawinski, K., Lintott, C., Thomas, D., et al. 2009, Am. Astron. Soc., 41, 277

Shapiro, K. L., Falcón-Barroso, J., van de Ven, G., et al. 2010, MNRAS, 402, 2140

Stasińska, G., Vale Asari, N., Cid Fernandes, R., et al. 2008, MNRAS, 391, L29

Stasińska, G., Costa Duarte, M. V., Vale Asari, N., Cid Fernandes, R., \& Sodré Jr., L. 2015, MNRAS, 449, 559

Trager, S. C., Faber, S. M., Worthey, G., \& González, J. J. 2000, AJ, 120, 165 Tremonti, C. A., Heckman, T. M., Kauffmann, G., et al. 2004, ApJ, 613, 898

Trinchieri, G., \& di Serego Alighieri, S. 1991, AJ, 101, 1647

York, D. G., Adelman, J., Anderson, Jr., J. E., et al. 2000, AJ, 120, 1579

1 Instituto de Astrofísica e Ciências do Espaço, Universidade do Porto, Centro de Astrofísica da Universidade do Porto, Rua das Estrelas, 4150-762 Porto, Portugal

e-mail: jean@astro.up.pt

2 Instituto de Astrofísica de Andalucía (CSIC), Glorieta de la Astronomía s/n Aptdo. 3004, 18080-Granada, Spain

3 Estación Experimental de Zonas Aridas (CSIC), Ctra. de Sacramento s.n., La Cañada, 04120 Almería, Spain

4 Institut d'Astrophysique de Paris, UMR 7095, CNRS, Université Pierre et Marie Curie, 98 bis boulevard Arago, 75014 Paris, France

5 Instituto de Astronomía,Universidad Nacional Autonóma de Mexico, A.P. 70-264, 04510, México, D.F., Mexico

6 University of Vienna, Türkenschanzstrasse 17, 1180 Vienna, Austria

7 Sydney Institute for Astronomy, University of Sydney, NSW 2006, Australia

8 Millennium Institute of Astrophysics, Chile

9 Departamento de Astronomía, Universidad de Chile, Casilla 36-D, 1058 Santiago, Chile

10 Astronomical Institute of the Ruhr-University Bochum, Universitätsstr. 150, 44580 Bochum, Germany

11 RUB Research Department "Plasmas with Complex Interactions", Universitätsstr. 150, 44580 Bochum, Germany

12 Instituto Nacional de Astrofísica, Óptica y Electrónica, Luis E. Erro 1, 72840 Tonantzintla, Puebla, Mexico

13 Leibniz-Institut für Astrophysik Potsdam (AIP), An der Sternwarte 16, 14482 Potsdam, Germany

14 CIEMAT, Avda. Complutense 40, 28040 Madrid, Spain

15 CEI Campus Moncloa, UCM-UPM, Departamento de Astrofísica y CC. de la Atmósfera, Facultad de CC. Físicas, Universidad Complutense de Madrid, Avda. Complutense s/n, 28040 Madrid, Spain

16 Department of Physics, Institute for Astronomy, ETH Zürich, 8093 Zürich, Switzerland

17 Departamento de Astrofísica y CC. de la Atmósfera, Universidad Complutense de Madrid, 28040 Madrid, Spain

18 Australian Astronomical Observatory, PO Box 915, North Ryde, NSW 1670, Australia

19 Department of Physics and Astronomy, Macquarie University, NSW 2109, Australia 